UCRL-ID-130839

\title{
TART97 Installation
}

\author{
D. E. Cullen
}

May 18, 1998

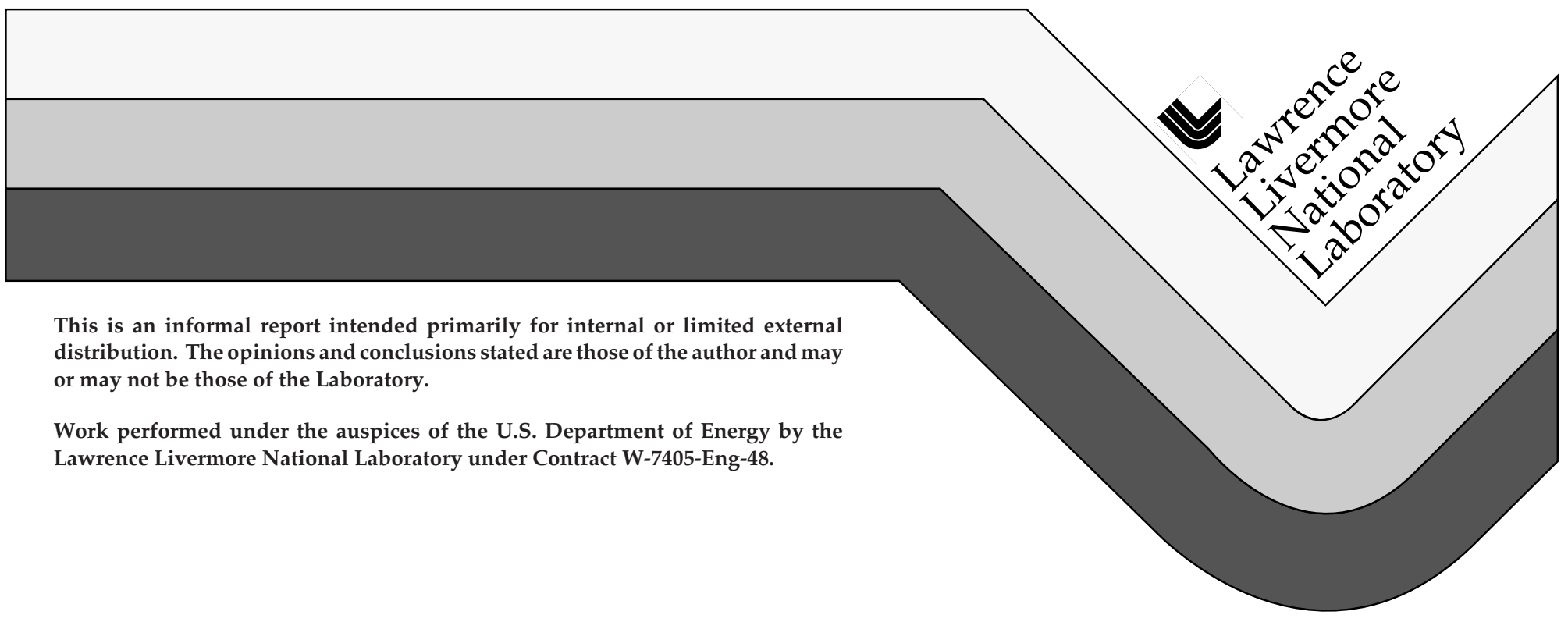




\section{DISCLAIMER}

This document was prepared as an account of work sponsored by an agency of the United States Government. Neither the United States Government nor the University of California nor any of their employees, makes any warranty, express or implied, or assumes any legal liability or responsibility for the accuracy, completeness, or usefulness of any information, apparatus, product, or process disclosed, or represents that its use would not infringe privately owned rights. Reference herein to any specific commercial product, process, or service by trade name, trademark, manufacturer, or otherwise, does not necessarily constitute or imply its endorsement, recommendation, or favoring by the United States Government or the University of California. The views and opinions of authors expressed herein do not necessarily state or reflect those of the United States Government or the University of California, and shall not be used for advertising or product endorsement purposes.

This report has been reproduced directly from the best available copy.

Available to DOE and DOE contractors from the Office of Scientific and Technical Information P.O. Box 62, Oak Ridge, TN 37831

Prices available from (423) 576-8401

Available to the public from the National Technical Information Service

U.S. Department of Commerce 5285 Port Royal Rd. Springfield, VA 22161 


\author{
TART97 Installation \\ b $\mathbf{y}$ \\ Dermott E. Cullen \\ University of California \\ Lawrence Livermore National Laboratory \\ P.O. Box 808 \\ L - 59 \\ Livermore, CA 94550 \\ e.mail: cullen1@llnl.gov \\ tele: 510-423-7359
}

\title{
Acknowledgments
}

I thank Stella Hadjimarkos, LLNL, for installing and testing the CRAY version of TART97. I thank Jennie Manneschmidt, Radiation Safety Information Computational Center (RSICC), Oak Ridge National Laboratory, for testing and correcting the preliminary version of TART97 CD. Jennie's efforts led to many significant corrections and improvements to the final distributed version of TART97 CD, which hopefully will make TART97 CD contain fewer errors and omissions, and be more user friendly. Thank you, Jennie, you did a SUPER JOB!

\section{What Computer Should You USE?}

I am not going to try to tell anybody how to do their job, but I will point out the advantages of using a 32 bit computer instead of a CRAY. If you have access to be variety of computers, the decision as to which you use is naturally strictly up to you. However, please consider the following points.

The CRAY is a great computer for running codes that can take advantage of its vector capabilities. However, TART97 is a scalar code, designed to run on virtually any computer, and it does not take full advantage of the CRAY's capabilities. If you read the TART97 documentation you will find a table comparing the running time of TART97 on a variety of computers. You will find from this table that there are now a variety of 32 bit computers that can run TART97 faster and much cheaper than a CRAY can. For example, even a Laptop computer runs TART97 twice as fast as a CRAY YMP, and an IBM-PC, $233 \mathrm{MHz}$ Pentium-II, runs TART97 six times as fast as a CRAY YMP. Remember, the times in this table for CRAY are CPU 
times. If many users are using the CRAY at the same time it can take much longer than the time given here until the actual time that your run finishes and you have the results. In contrast for the 32 bit systems the times in this table are roughly the time until you actually have the results. Therefore if you factor in the cost of your salary while you are waiting for results, the advantage of 32 bit computers over the CRAY is even larger than indicated in this table.

A second point to consider is that TART97 is a complete system, that will help you prepare and verify TART97 input, and analyze TART97 output results. Using the entire system can save you enormous amounts of time and energy. However, you will only be able to use the entire system on 32 bit computers, i.e., the interactive graphics codes only work on 32 bit computers, not on a CRAY. Even if you do decide to use a CRAY to run TART97, I strongly recommend that you implement the remainder of the system on a 32 bit system, e.g., UNIX workstation, IBM-PC, or POWERMAC. Believe me when I say, having these codes available will save you enormous amounts of time and energy, and minimize your frustration when preparing input and analyzing output.

\section{Overview}

This installation manual is divided into six parts,

1) An Overview of TART97 CD and what all users should know

2) IBM-PC

3) POWERMAC

4) CRAY

5) UNIX - including all variants of UNIX, e.g., LINUX, etc.

6) OTHER computers

The first part should be read by all users. The remaining parts are relevant only to users of each specific type of system. For users of OTHER types of computers, first read the section on OTHER, that explains computer dependence, then read the UNIX section.

\section{Format of TART97 CD}

This CD is written in the ISO 9660 format, which is designed to allow a $\mathrm{CD}$ to be read on any computer. As applied to TART97 CD, there are several important limitations of the ISO 9660 format including, 
1) Filenames are limited to the DOS 8.3 convention (names up to 8 characters in length, optionally followed by an extension of up to 3 characters). Fortunately the TART97 system already conforms to this limitation,

2) This format cannot distinguish between upper and lower case characters. Since TART97 CD uses upper and lower case filenames, this could present an initial problem during installation.

I have attempted to minimize the impact of this limitation on users by supplying compressed, combined files for use on all computers (IBM-PC, POWERMAC, CRAY, and UNIX). When uncompressed and installed these files will expand to properly define all of the filenames used by TART97.

The only exceptions will be the compressed file, TART97.tar, and installation file, INSTALL.BAT, that is included for CRAY and UNIX. When you copy these files from the TART97 CD the names may end up on your computer in lower case (install.bat), upper case (INSTALL.BAT), or a mix of the two. In the following installation instructions I will assume these files are named TART97.tar and INSTALL.BAT. If they are not, please change them to this.

\section{Reading TART97 CD}

A commercially produced $\mathrm{CD}$ has a slightly different reflectivity, when compared to a TART97 CD that I produce on my IBM-PC at home. This may initially cause your system to have a problem reading TART97 $\mathrm{CD}$. If it does, open your $\mathrm{CD}$ drive, jiggle the $\mathrm{C}$ around a little to re-position it, and then try again. It generally doesn't take more than a few tries until you can read TART97 CD, and once you read it, you are finished using it.

\section{Contents of TART97 CD}

This CD contains,

\section{Level 1: Computer Systems}

The CD includes on-line documentation and the system of codes for a variety of computers. For a specific computer (e.g., IBM, POWERMAC, etc.) you ONLY need ALL of the files in the directory for that computer, 
IBMPC

POWERMAC

CRAY

UNIX

ALL users should have a copy of the documentation. On-line documentation is in Microsoft Word format created on an IBM-PC, but can be read by most word processing codes. A MAC formatted version of the documentation is included with the MAC installation file.

For IBM-PC and POWERMAC EXECUTABLE codes are provided. In both cases compressed, combined files are provided, that can be easily installed and immediately used.

Since there are a variety of CRAY computers and UNIX workstations, SOURCE codes and installation instructions are provided. In this case a compressed, combined file is provided, that can be used to easily install everything. Using the compressed, combined files requires using UNIX tar uncompress. The UNIX directory should be used for all variants of UNIX, e.g., LINUX, etc.

For OTHER computers, it is recommended that you use the combined file from the UNIX directory.

\section{Level 2: Documentation and The Code System}

For each type of computer you will find sub-directories,

Documentation, Production Codes and input data

DOCUMENT - On-line documentation

TART97 - Execute TART97

UTILITY - A collection of executable utility codes

EXAMPLES - Example TART97 input files

Interactive Graphics Codes (not included for CRAY)

TARTCHEK - Check TART input, and overlay results on geometry EPICSHOW - Plot Neutron, Photon, Electron and Charged Particle data PLOTTAB - General plotting code used by utility codes EDITOR - FORTRAN and TART input editor 
CRAY and UNIX users should have two additional sub-directories,

TARTDATA - Create TART97 binary data files

EPICDATA - Create EPICSHOW binary data files

These two directories can be deleted once installation is completed.

WARNING - There are files in several of these directories that have the same name - therefore be sure that you keep ALL of these files in separate directories.

\section{Recommended Directory Structure}

The installation instructions explain how to set up a directory structure during installation, so you need not do this before hand. It is mentioned here only to encourage you to use the Recommended Directory Structure during installation.

It is HIGHLY RECOMMENDED that you set up a directory structure to include all parts of this system in a directory named TART97. The INSTALL.BAT files will automatically uncompress everything assuming the recommended directory structure, including the subdirectories named above.

PLEASE use this recommended directory structure to insure that future updates to this system can be easily added to your computer.

\section{Level 3: Neutron and Photon Data}

Standard data files included in this package are identified as ENDL and include,

1) 175 group neutron data derived using the Livermore ENDL file. For compatibility with older TART libraries only PROMPT NU-BAR, not TOTAL NU-BAR is used in this file.

2) 176 point photon data derived using the Livermore EPDL97 file.

Alternative data files included in this package are identified as ENDFENDL and include,

1) 650 group neutron data derived using a combination of ENDF/BVI and ENDL data. TOTAL NU-BAR is used in this file. The new 650 
group structure extends up to $1 \mathrm{GeV}$. Presently nuclear data is only available up to $20 \mathrm{MeV}$, so that only 566 of the groups are currently used.

2) 176 point photon data derived using the Livermore EPDL97 file.

Since the ENDF/B-VI photon interaction data file is based on the Livermore EPDL97 file, there is no need to have separate TART97 photon data files based on the Livermore EPDL file and the ENDF/BVI file - they are the same.

The older 175 group neutron data is provided for users whose applications may in some way be dependent on using the older TART group structure, e.g., you have codes that process the output assuming 175 group results.

The newer 650 group neutron data is provided for all other users. I strongly recommend that if you are not in some way dependent on the TART 175 group structure, use the newer 650 group data.

\section{General Information}

\section{Recommended Filenames}

When using TART97 you can optionally input filenames for input parameters and the output report, see TART95.DOC. However it is STRONGLY RECOMMENDED that you only use the default filenames TART.IN and TART.OUT for input and output. All of the other codes in the TART97 system use these standard filenames, and you will find it is much easier to use the entire system if you use these default filenames.

\section{Moving Files between Computers}

All of the files on this $C D$ have been written as native binary for each type of computer. If you move any of these files between computers you MUST move them as BINARY.

Because of the LOWER and UPPER case filename conventions used by TART97 if you move files between case insensitive and case sensitive computers, e.g., copying TART.IN from an IBM-PC to UNIX computer you will end up with a file named tart.in - in which case you should rename it to TART.IN. 
OVERVIEW

\section{The Use of Binary, Random Access Files}

WARNING - The procedures used to produce and use binary, random access files is EXACTLY the same on EVERY computer. However, the physical layout of the actual data files varies from one computer to another. THEREFORE UNDER NO CIRCUMSTANCE SHOULD YOU TRY TO USE THE BINARY FILES PRODUCED ON ONE TYPE OF COMPUTER ON A DIFFERENT TYPE OF COMPUTER. Separate binary files MUST be created on EACH type of computer that you use.

\section{System Wide Installation}

If you wish you can install TART97 so that it is accessible to everyone on your system, without the necessity of their having their own copy of the code and its data files.

By placing TART97 and its 4 data files in a common directory and defining paths most computers are smart enough to search this common directory for both TART97 and its data files. If you do, users will ONLY be able access ONE set of data files - either ENDL or ENDFENDL - you can decide which.

If your computer isn't smart enough to find the 4 data files, in TART97 see, SUBROUTINE OPENTART for instructions on how to add a path to your common directory to find the four data files. From SUBROUTINE OPENTART you will see that you can add a path to the data files - to only ONE of the two sets of data files - either ENDL or ENDFENDL - you can decide which.

If you do this TART97 will: 1) first search for the file in the current directory, 2) if it isn't found, next search using the path you have defined.

If you do set up TART97 for system wide use using ONE of the two available set of data files, I suggest that you make the other set of the data accessible on your system, so that users who wish can copy the alternate set of data files into their directory and use it, i.e., TART97 will automatically use the files in the user's directory, rather than the files that you have set up for system wide use.

\section{The Next Level}

Once you have selected the codes for your type of computer, i.e., the 
directory IBMPC, POWERMAC, CRAY, or UNIX, see the part of this installation manual that applies to your type of computer.

\section{Register as a User}

It is important that you register as a user of this system, so that you are included on our distribution list for newsletters and code updates.

If you have access to e.mail send your e.mail address and complete postal mailing address to me at,

\section{cullen1@1lnl.gov}

If you do not have access to e.mail send your complete postal mailing address to me at the above address.

\section{You can HELP}

You can help in several ways,

1) Improvements to systems such as this depend heavily on user feedback. If you find any errors in the documentation or codes, or have suggestions for improving the system, please inform me, preferably by e. mail.

Sending me complete documents or codes saying you have "fixed" them is a waste of your time and mine. Usually when there is an error many users will find it, and if they all send me "fixed" versions, I have no idea which of them is the "best fix", and more importantly I have no idea if the "fix" works on every computer. Before including any "fixes" I have to decide what is the "best fix" from the viewpoint of the entire TART97 system and its use on many different types of computers.

Please keep your corrections as short as possible, e.g., a sentence or two from documents, a few lines from codes - in either case including both the original and your suggested changes.

2) The TART97 CD is not made at some high tech facility, neither is it made for me by the Easter Bunny. It is made by me, at home, on $\mathrm{my}$ own time, on my IBM-PC. It is a time consuming process to make each copy of TART97 CD. 
Therefore, after you have copied everything that you need from the $\mathrm{CD}$, you can help by returning it to me so that I can pass it on to the next person who requests it. As an added incentive I'll mention that those who return the $\mathrm{CD}$ to me are placed at the head of the list for updates. 


\section{IBM-PC}

\section{2 versus 16 bit Systems}

The distributed TART97 system is designed to run on 32 bit systems (Windows'95 or Windows/NT), not 16 bit systems (Windows 3.x or DOS). On exactly the same IBM-PC a 32 bit system will run programs, such as TART97, over twice as fast as on 16 bit systems. In addition the 32 bit systems are reasonably priced (less than \$100). Therefore I highly recommend that if you are not using a 32 bit system, you update your system; this is a very inexpensive means of more than doubling the power of your IBM-PC.

\section{Installation and Testing of Codes}

1) Make sure you do not have a directory named TART97, otherwise installation will overwrite it.

2) Using Windows, select the drive to install TART97 on, i.e., drive C:, D:, etc.

3) Use your RIGHT mouse key to drag and drop TART97.EXE and INSTALL.BAT from the TART97 CD IBMPC directory to your hard disk drive, i.e., drive C:, D:, etc.

4) To install everything double click on INSTALL.BAT. WARNING DO NOT double click on TART97.EXE - if you do, the correct directory and file structure will NOT be installed - ONLY use INSTALL.BAT for installation. It will take a few minutes to install everything.

5) Once installation is finished you can delete INSTALL.BAT and TART97.EXE.

That's it - installation is complete.

\section{Installed Directory Structure}

You should now have a main directory named TART97, and within this directory you should have the following sub-directories,

\section{Documentation}

DOCUMENT - On-line documentation in Microsoft Word format

\section{Production Codes}


TART97 - TART97 source code

UTILITY - A variety of useful utility codes

EXAMPLES - Example TART97 input parameters

\section{Graphics Codes}

TARTCHEK - TARTCHEK source code

EPICSHOW - EPICSHOW source code

PLOTTAB - PLOTTAB source code

EDITOR - FORTRAN code editor

All of the codes are in executable form, so you can immediately start using the codes.

\section{Verification}

It is highly recommended that you not be in too much of a rush to start using the system, and instead spend the time to FIRST verify that TART97 is producing reliable results.

The same example problem is in both ENDL and ENDFENDL directories ready to be used with TART97. It is HIGHLY RECOMMENDED that you take the time to first run this problem in at least one, and preferable both of these directories. In either directory to start the problem

double click on TART97-5.

Execution time will be somewhere between 1,000 and 18,000 seconds, (about 20 minutes to 5 hours), depending on the speed of your computer. When this problem ends, copy the utility code, critedit, from the utility directory to the current directory and execute critedit by double clicking on it.

This will give you a summary of the results of the 68 problems run. The important things to check are at the bottom of the output listing, on your screen, and in the file CRITEDIT.LST.

First check the running time, which will give you a good indication of the relative power of your computer - see TART97.DOC for a list of expected times on a wide variety of computers.

Next check the calculated Average K-eff - this should be about, 
$\mathrm{ENDL} \quad=0.994$ to 0.995

ENDFENDL $=0.999$ to 1.000

The $0.5 \%$ difference is because ENDL is using PROMPT NU-BAR and ENDFENDL is using the more realistic TOTAL NU-BAR.

If you do not get results similar to these, go no further - something is wrong - notify the author.

If your results look o.k., that's it - installation and verification are completed - HAVE FUN!!! 


\section{Power MAC}

\section{Installation and Testing of Codes}

The MAC file system is different from other file systems, in the sense that for each file that you can "see" it uses hidden files to define status information. This makes it impossible to transfer files in their original form between MAC and other computers, as had to be done to create TART97 CD.

This difficulty has been overcome by compressing the entire system into a single Self Extracting Archive file, and then using Stuffit Deluxe to produce a single file, including all of the hidden files.

In order to implement this system you have do these two steps in reverse: first use Stuffit Expander to create the Self Extracting Archive file, and then uncompress to install the entire system. If you do not have a copy of Stuffit Expander, it is available FREE on the web at,

http://www.aladdinsys.com/consumer/index.html

Once you have Stuffit Expander, to install the entire system,

1) Make sure you do not have a directory named TART97, otherwise installation will overwrite it.

2) Copy TART97.sit from the TART97 CD POWERMAC directory to your desk top

3) Use Stuffit Expander to expand TART97.sit.

4) This will create the Self Extracting Archive file TART97.sea.

5) You no longer need TART97.sit and you can delete it.

6) Double click on TART97.sea to start uncompressing.

7) Direct it to uncompress everything into a directory named TART97 (the default).

8) Everything should now be installed in sub-directories within TART97.

9) You no longer need TART97.sea and you can delete it.

That's it - installation is complete.

\section{Installed Directory Structure}

You should now have a main directory named TART97, and within 
this directory you should have the following sub-directories,

\section{Documentation}

DOCUMENT - On-line documentation in Microsoft Word format

\section{Production Codes}

TART97 - TART97 source code

UTILITY - A variety of useful utility codes

EXAMPLES - Example TART97 input parameters

\section{Graphics Codes}

TARTCHEK - TARTCHEK source code

EPICSHOW - EPICSHOW source code

PLOTTAB - PLOTTAB source code

EDITOR - FORTRAN code editor

All of the codes are in executable form, so you can immediately start using the codes.

\section{Verification}

It is highly recommended that you not be in too much of a rush to start using the system, and instead spend the time to FIRST verify that TART97 is producing reliable results.

The same example problem is in both ENDL and ENDFENDL directories ready to be used with TART97. It is HIGHLY RECOMMENDED that you take the time to first run this problem in at least one, and preferable both of these directories. In either directory to start the problem

double click on TART97.5.

Execution time will be somewhere between 1,000 and 18,000 seconds, (about 20 minutes to 5 hours), depending on the speed of your computer. When this problem ends, copy the utility code, critedit, from the utility directory to the current directory and execute critedit by double clicking on it.

This will give you a summary of the results of the 68 problems run. 
The important things to check are at the bottom of the output listing, on your screen, and in the file CRITEDIT.LST.

First check the running time, which will give you a good indication of the relative power of your computer - see TART97.DOC for a list of expected times on a wide variety of computers.

Next check the calculated Average K-eff - this should be about,

ENDL $\quad=0.994$ to 0.995

ENDFENDL $=0.999$ to 1.000

The $0.5 \%$ difference is because ENDL is using PROMPT NU-BAR and ENDFENDL is using the more realistic TOTAL NU-BAR.

If you do not get results similar to these, go no further - something is wrong - notify the author.

If your results look o.k., that's it - installation and verification are completed - HAVE FUN!!! 


\section{UNIX}

\section{2 versus 64 bit Computers}

On all computers TART97 uses 64 bit precision for REAL variables. 64 bit precision means DOUBLE PRECISION on 32 bit UNIX workstations, and SINGLE PRECISION on 64 bit CRAY computers. The only difference between the UNIX and CRAY versions of TART97 is that the UNIX version uses DOUBLE PRECISION functions, e.g., DEXP, DCOS, DABS, etc., and the CRAY version uses SINGLE PRECISION functions, e.g., EXP, COS, ABS, etc.

WARNING - because of this difference the UNIX and CRAY versions are not interchangeable between computers. On UNIX computers, ONLY use the UNIX version, and on CRAY computers, ONLY use the CRAY version.

\section{Installation and Testing of Codes}

1) Make sure you do not have a directory named TART97, otherwise installation will overwrite it.

2) Copy TART97.tar and INSTALL.BAT from the TART97 CD UNIX directory to your computer. Make sure the names are EXACTLY as shown here.

3) Make sure INSTALL.BAT is executable,i.e., type, chmod 777 INSTALL.BAT

4) Execute INSTALL.BAT.

5) Once installation is finished you can delete INSTALL.BAT and TART97.tar.

\section{Installed Directory Structure}

You should now have a main directory named TART97, and within this directory you should have the following sub-directories,

\section{Documentation}

DOCUMENT - On-line documentation in Microsoft Word format

\section{Production Codes}

TART97 - TART97 source code

UTILITY - A variety of useful utility codes 
TARTDATA - Codes and data to create TART97 data files

EXAMPLES - Example TART97 input parameters

\section{Graphics Codes}

TARTCHEK - TARTCHEK source code

EPICDATA - Codes and data to create EPICSHOW data files

EPICSHOW - EPICSHOW source code

PLOTTAB - PLOTTAB source code

EDITOR - FORTRAN code editor

Here's a brief outline of what you have to do to implement and the verify the system,

\section{Production Codes}

1) Create the binary, random access files used by TART97

2) Compile TART97

3) Compile the utility codes

\section{Interactive Graphics Codes}

4) Create the binary, random access files used by EPICSHOW

5) Compile and load TARTCHEK, EPICSHOW, PLOTTAB, and EDITOR

\section{Verification}

6) Run the TART97 benchmark problem

\section{Compilation Errors and Warning Messages}

While compiling ALL of the codes you should not receive ANY compiler Error messages. While compiling TART97 you should receive a few Warnings that certain parts of the code cannot be reached - this is o.k. - these are parts of the code that are currently inactive; see the TART95.DOC for details. On some computers you will get MANY Warning messages that some variables are not being used - this is o.k. - your compiler is merely listing all of the variables from the INCLUDE files that may not be used by individual routines.

If you receive any other Error or Warning messages please report them to me in detail. 


\section{Optimization Level}

Depending on how your computer is configured you may receive compiler/loader messages that compilation or loading is aborted due to exceeding your systems resources; this message is stated in different ways on various computers, but the intent on all computers is the same. There are several ways to correct this problem: a) The simplest way is to decrease the optimization level in your Makefile, e.g., change -O5 to -O2. This is not recommended, since reducing optimization will increase execution time, b) Have your system supervisor increase the system resources allowed for compilation/loading. This is the recommended procedure, but may not be practical if nobody at your installation knows how to do this.

\section{UNIX vs. Other System Users}

The following instructions are for UNIX users; this includes all variants of UNIX, such as LINUX, etc. For users of other systems first see the instructions in the OTHER section of this installation manual, for information on computer dependence. You can then return to this point and follow these steps - modified to meet your computer's needs.

Here are the details of the steps to follow,

\section{Production Codes}

1) In TARTDATA there are separate directories for the ENDL and ENDFENDL data files - I recommend that you implement both of them and then based on your usage decide which you want to continue to use.

In either directory ENDL or ENDFENDL make sure the file MAKEIT.BAT is executable, i.e., type,

chmod 777 MAKEIT.BAT

Then execute it by typing,

\section{MAKEIT.BAT}

This will compile four codes and then run each code to create the four binary, random access files used by TART97. 
On most computers there is nothing to do until this batch file finishes running. However, on some computers you will be asked to approve deletion of certain files - answer yes (y) each time.

When this batch file has finished running you will find the four random access, binary files,

TARTND - Neutron interaction data

TARTPPD - Neutron induced photon production data

GAMDAT - Photon interaction data

NEWCROSS - Neutron multi-band, self-shielding data

Move these four files to the directory where you will execute TART97 - either the directory /TART97/TART97/ENDL or /TART97/TART97/ENDFENDL, depending on which data files you created.

Once you have created the binary, random access data files and moved them to where you will be using them you no longer need the TARTDATA directory and you can delete it.

2) In TART97, you next have to compile TART97 in the directory TART97/TART97/SOURCES. There are still a few computer dependent parts of TART97, e.g., execution time, date, etc. All of these computer independent parts are isolated in files named ???.f - where ??? is the name of a specific type of computer, e.g., SUN.f, HP.f, etc.

Makefiles are provided for each type of computer. The only differences between the various Makefiles are: a) they will use the correct ???.f file for each type of computer, b) they will compile on each computer using the highest level of optimization defined by each computer. The Makefiles use a similar name convention, e.g., SUN.mak, HP.mak, etc.

To compile all you need do is select the Makefile for your computer, copy it to Makefile, e.g., for use on a SGI,

cp SGI.mak Makefile

Make sure Makefile is executable and then make the executable by typing, 
make

Next copy tart97.5 to the TART97/TART97/ENDL and TART97/TART97/ENDFENDL sub-directories where you will be executing the code.

Once you have done this you no longer need the source code, Makefiles, etc. in this directory and you can delete the directory TART97/TART97/SOURCES.

When you have completed this step you will be able to run TART97.5 using either the ENDL or ENDFENDL data. In either of these directories to run the example problem type,

$\operatorname{tart97.5}$

3) In UTILITY, make sure the file INSTALL.BAT is executable, e.g., chmod 777 INSTALL.BAT

Then execute it by typing, INSTALL.BAT

This will compile all of the utility codes for your later use. See the documentation of the utility codes for details on how to use the utility codes.

Once you have done this you no longer need the source code, in this directory and you can delete all *.f and INSTALL.BAT.

\section{Interactive Graphics Codes}

\section{2 versus 64 bit}

The interactive graphics codes are only designed to be used on 32 bit computers. If you are using a CRAY you will not be able to use these codes. However, these codes offer such advantages that even if your primary computer is a CRAY, if you have access to any UNIX workstations, IBM-PC, or POWERMAC I highly recommend that you implement these codes. These codes can save you an enormous amount of time and energy in preparing and testing TART97 input, and in analyzing TART97 output. 


\section{2 bit}

In principle, for these codes you will be able to use simple procedures similar to those used above to install TART97. In practice interactive graphics are still not completely standard and you may have more work to do. Since any problems that you may encounter apply to all of the following codes let go over them once here - once you solve these problems for one of the codes, all the others will be simple plug-ins. Here's a list of the problems you may run into and how to solve them,

1) The codes use the standard UNIX X11 graphics library. This is a standard library, but it isn't always stored in the same place on all computers - so it may not be where the enclosed Makefiles are looking for it. Therefore you may have to modify the Makefiles to direct them to where X11 is stored on your system - or alternatively, copy the X11 library to where the

Makefiles are looking for them.

2) The graphics interface is written in $\mathrm{C}$ and the FORTRAN to $\mathrm{C}$ interface is not the same on all computers. On some computers all $\mathrm{C}$ routine names end in underscore (_), while on others they do not. Routines are supplied to handle both conventions,

dash.c = includes - at the end of names

nodash.c $=$ does not include

If during loading you receive error messages that many routines are missing, look in the Makefile you are using to see whether you are using either, dash.c or nodash.c - and try using the other one.

3) Lastly in the case of TARTCHEK you should define running time. If you have gotten this far you have already gotten TART97 running, including a timing routine - so you can use the same procedure here. The only difference is that TART97 defines time since the beginning of execution, whereas TARTCHEK defines time between calls to the timing routine - for details, see the timer routines in the TARTCHEK directory.

Solving these problems may not be that simple - particularly the problem of finding the X11 graphics system on your computer and modifying the Makefiles to access X11. However, be assured that this 
system has been installed on many, many UNIX systems and we have NEVER found one that we couldn't implement these codes on. Some systems are more difficult than others, but eventually we have gotten the codes to work ON ALL OF THEM! So hang in there and keep trying - it can be done, and having these codes available is worth the effort NOW, because they will make your life a lot easier in the FUTURE.

How do you find out if you have any of these problems? Try compiling TARTCHEK and you'll find out rather quickly.

Below I assume you have solved all of these problems and I will discuss implementation of the remaining codes.

4) In EPICDATA, make sure the file INSTALL.BAT is executable, e.g., chmod 777 MAKEIT.BAT

Then execute it by typing,

\section{INSTALL.BAT}

This will compile a code and then run it to create the four, binary, random access files used by EPICDATA.

On most computers there is nothing to do until this batch file finishes running. However, on some computers you will be asked to approve deletion of certain files - answer yes (y) each time.

When this batch file has finished running you will find the four random access, binary files,

NEUTRON.BIN - Neutron interaction data

PHOTON.BIN - Photon interaction data

ELECTRON.BIN - Electron interaction data

CHARGED.BIN - Light Charged Particle interaction data

Move these four files to the directory where you will execute EPICSHOW, e.g., /TART97/EPICSHOW.

Once you have created the binary, random access data files and moved them to where you will be using them you no longer need the EPICDATA directory and you can delete it. 
5) The procedure for implementing the graphics codes is the same for all four graphics codes,

\section{TARTCHEK}

EPICSHOW

\section{PLOTTAB}

EDITOR

There is a directory for each, and within each of these directories there is a sub-directory named SOURCES. As with TART97, these directories contain the basic source code, plus computer dependent code for each type of computer, and Makefiles for each type of computer. In each case all you have to do is select the Makefile for your type of computer, copy it to Makefile, e.g., for use on an SGI,

cp SGI.mak Makefile

and then compile and load by typing,

make

In the case of PLOTTAB, there are two versions of the code, plottab, the interactive graphics version for on screen results, which will be created using the above instructions, and plotsave, the noninteractive version that for Postscript formatted results. Plotsave is completely computer independent and will be created by executing,

PLOTSAVE.mak

Once you have compiled and loaded a code, move it from the SOURCES directory, e.g., for tartchek, move it from TARTCHEK/SOURCES to TARTCHEK. In the case of PLOTTAB remember to move both plottab and plotsave. When you have done this you no longer need the source code, Makefiles, etc. and you can delete the SOURCES directory.

\section{Verification}

6) It is highly recommended that you not be in too much of a rush to start using the system, and instead spend the time to FIRST verify that TART97 is producing reliable results. 
The same example problem is in both ENDL and ENDFENDL directories ready to be used with TART97. It is HIGHLY RECOMMENDED that you take the time to first run this problem in at least one, and preferable both of these directories. In either directory to start the problem type,

$\operatorname{tart97.5.}$

Execution time will be somewhere between 1,000 and 18,000 seconds, (about 20 minutes to 5 hours), depending on the speed of your computer. When this problem ends, copy the utility code, critedit, from the utility directory to the current directory and execute critedit by typing,

critedit

This will give you a summary of the results of the 68 problems run. The important things to check are at the bottom of the output listing, on your screen, and in the file CRITEDIT.LST.

First check the running time, which will give you a good indication of the relative power of your computer - see TART97.DOC for a list of expected times on a wide variety of computers.

Next check the calculated Average K-eff - this should be about,

$\mathrm{ENDL} \quad=0.994$ to 0.995

ENDFENDL $=0.999$ to 1.000

The $0.5 \%$ difference is because ENDL is using PROMPT NU-BAR and ENDFENDL is using the more realistic TOTAL NU-BAR.

If you do not get results similar to these, go no further - something is wrong - notify the author.

If your results look o.k., that's it - installation and verification are completed - HAVE FUN!!! 


\section{CRAY}

\section{2 versus 64 bit Computers}

On all computers TART97 uses 64 bit precision for REAL variables. 64 bit precision means DOUBLE PRECISION on 32 bit UNIX workstations, and SINGLE PRECISION on 64 bit CRAY computers. The only difference between the UNIX and CRAY versions of TART97 is that the UNIX version uses DOUBLE PRECISION functions, e.g., DEXP, DCOS, DABS, etc., and the CRAY version uses SINGLE PRECISION functions, e.g., EXP, COS, ABS, etc.

WARNING - because of this difference the UNIX and CRAY versions are not interchangeable between computers. On UNIX computers, ONLY use the UNIX version, and on CRAY computers, ONLY use the CRAY version.

\section{CRAY UNICOS}

The CRAY version of TART97 is configured to compile and run under CRAY UNICOS. If you are using a different CRAY system, see the section on OTHER computers.

\section{Installation and Testing of Codes}

1) Make sure you do not have a directory named TART97, otherwise installation will overwrite it.

2) Copy TART97.tar and INSTALL.BAT from the TART97 CD CRAY directory to your computer. Make sure the names are EXACTLY as shown here.

3) Make sure INSTALL.BAT is executable,i.e., type, chmod 777 INSTALL.BAT

4) Execute INSTALL.BAT.

5) Once installation is finished you can delete INSTALL.BAT and TART97.tar.

\section{Installed Directory Structure}

You should now have a main directory named TART97, and within this directory you should have the following sub-directories,

\section{Documentation}


DOCUMENT - On-line documentation in Microsoft Word format

\section{Production Codes}

TART97 - TART97 source code

UTILITY - A variety of useful utility codes

TARTDATA - Codes and data to create TART97 data files

EXAMPLES - Example TART97 input parameters

\section{Graphics Codes}

These are not provided for CRAY. To use them you will have to use a UNIX workstation, IBM-PC or POWERMAC.

Here's a brief outline of what you have to do to implement and the verify the system,

\section{Production Codes}

1) Create the binary, random access files used by TART97

2) Compile TART97

3) Compile the utility codes

\section{Verification}

4) Run the TART97 benchmark problem

\section{Compilation Errors and Warning Messages}

While compiling ALL of the codes you should not receive ANY compiler Error messages. While compiling TART97 you should receive a few Warnings that certain parts of the code cannot be reached - this is o.k. - these are parts of the code that are currently inactive; see the TART95.DOC for details. On some computers you will get MANY Warning messages that some variables are not being used - this is o.k. - your compiler is merely listing all of the variables from the INCLUDE files that may not be used by individual routines.

If you receive any other Error or Warning messages please report them to me in detail.

Here are the details of the steps to follow, 


\section{Production Codes}

1) In TARTDATA there are separate directories for the ENDL and ENDFENDL data files - I recommend that you implement both of them and then based on your usage decide which you want to continue to use.

In either directory ENDL or ENDFENDL, edit MAKEIT.BAT to use your compiler, then make sure the file MAKEIT.BAT is executable, e.g.,

chmod 777 MAKEIT.BAT

Then execute it by typing,

\section{MAKEIT.BAT}

This will compile four codes and then run each code to create the four binary, random access files used by TART97.

On most computers there is nothing to do until this batch file finishes running. However, on some computers you will be asked to approve deletion of certain files - answer yes (y) each time.

When this batch file has finished running you will find the four random access, binary files,

TARTND - Neutron interaction data

TARTPPD - Neutron induced photon production data

GAMDAT - Photon interaction data

NEWCROSS - Neutron multi-band, self-shielding data

Move these four files to the directory where you will execute TART97 - either $\quad$ /TART97/TART97/ENDL or /TART97/TART97/ENDFENDL, depending on which data files you created.

Once you have created the binary, random access data files and moved them to where you will be using them you no longer need the TARTDATA directory and you can delete it.

2) In TART97/SOURCES, you next have to compile TART97. TART97 is a single file that you should compile and load. The only special library used by TART97 is lmath. Compile using your compiler and 
name the executable tart97.5.

Next copy tart97.5 to the ENDL and ENDFENDL sub-directories where you will be executing the code.

Once you have done this you no longer need the source code, in this directory and you can delete the entire TART97/SOURCES directory.

When you have completed this step you will be able to run TART97 using either the ENDL or ENDFENDL data. In either of these directories to run the example problem type,

$\operatorname{tart} 97.5$

$3)$ In UTILITY, edit MAKEIT.BAT to use your compiler, make sure the file MAKEIT.BAT is executable, e.g.,

chmod 777 MAKEIT.BAT

Then execute it by typing,

\section{MAKEIT.BAT}

This will compile all of the utility codes for your later use. Once you have done this you no longer need the source code, in this directory and you can delete all *.f and MAKEIT.BAT.

\section{Verification}

4) It is highly recommended that you not be in too much of a rush to start using the system, and instead spend the time to FIRST verify that TART97 is producing reliable results.

The same example problem is in both ENDL and ENDFENDL directories ready to be used with TART97. It is HIGHLY RECOMMENDED that you take the time to first run this problem in at least one, and preferable both of these directories. In either directory to start the problem type,

$\operatorname{tart97.5.}$

Execution time will be somewhere between 1,000 and 18,000 seconds, (about 20 minutes to 5 hours), depending on the speed of 
your computer. When this problem ends, copy the utility code, critedit, from the utility directory to the current directory and execute critedit by typing,

critedit

This will give you a summary of the results of the 68 problems run. The important things to check are at the bottom of the output listing, on your screen, and in the file CRITEDIT.LST.

First check the running time, which will give you a good indication of the relative power of your computer - see TART97.DOC for a list of expected times on a wide variety of computers.

Next check the calculated Average K-eff - this should be about,

ENDL $\quad=0.994$ to 0.995

ENDFENDL $=0.999$ to 1.000

The $0.5 \%$ difference is because ENDL is using PROMPT NU-BAR and ENDFENDL is using the more realistic TOTAL NU-BAR.

If you do not get results similar to these, go no further - something is wrong - notify the author.

If your results look o.k., that's it - installation and verification are completed - HAVE FUN!!! 


\section{OTHER Users}

\section{Overview}

TART97 is so computer independent that you will find it is almost trivial to install and use it on any type of computer. Here I will describe in detail the few remaining computer dependent parts of the code. Once you have supplied these for your type of computer you can proceed to install using either the UNIX (32 bit computer) or CRAY (64 bit computer) installation instructions.

\section{Computer Dependence}

The only capability that you MUST have to use TART97 is CRAY like POINTERS, e.g.,

\section{POINTER(LOCX,X)}

to assign dynamic memory. If you don't have this capability, sorry about that, but you cannot use TART97.

TART97 is written in very strict FORTRAN and you should be able to use it on virtually any computer. The few remaining parts of the code are isolated in the module ???.f, where ??? defines each type of computer, e.g., SUN.f, HP.f. These features include, assignment of dynamic memory, defining time and date, and reading parameters from the input line; nothing else. Of these the only one that is REQUIRED is assignment of dynamic memory. As will be explained below, all of the others can be satisfied by dummy routines.

In order to implement the code on other computers you should be aware of the following computer dependence in ???.f; you can then define the equivalent for your computer and implement the system. I recommend that you start with SUN.f and modify it to meet your needs. There are only four computer dependent routines in each of the ???.f files.

\section{For Starters}

Try using SUN.f without any changes. If everything loads o.k. you are finished. However, if your loader tells you that any routines are missing you can use the following guidelines to define the missing 
routines.

As you will see below, the only necessary routine is the memory manager. All the others can be replaced by dummy routines (described in detail below). However, it is HIGHLY RECOMMENDED that you include a timing routine, so that you know how long the code takes to execute. EVERY computer has a means of defining execute, or real, running time; see the various ???.f files for how this is done on a variety of computers. For more details see more extensive remarks on timing at the end of this section.

\section{Subroutine MEMLOW}

Memory manager $=32$ vs. 64 bit pointers: so far the only workstation that we have found that uses 64 bit pointers is the DECAlpha. So in most cases you can use the routine from SUN.f. If your computer does use 64 bit pointers use the routine from DEC.f. If you are not sure how your computer treats pointers, try the 32 bit version from SUN.f.

For dynamic memory allocation this routine uses MALLOC, to assign memory, and FREE, to free memory. If the names of these routines are different on your computer, you need merely change MALLOC and FREE to the names used on your computer.

If your system does not have the equivalent of MALLOC and FREE, use the MALLOC.f routine supplied with the UNIX version.

Note, of the routines discussed here this is the only one that MUST be defined for TART97 to run properly.

\section{Subroutine INLINE}

Reads the input command line to optionally define the names of the TART input and output files. If your computer does not have a way to do this - no problem - just add a dummy routine to immediately return. This will limit you to ALWAYS using the default input and output filenames TART.IN and TART.OUT, which is what I recommend anyway.

SUBROUTINE INLINE(TARTIN,TARTOUT)

RETURN

END 


\section{Subroutine TIMEIT}

Define the execution time since the problem started running. Every computer has a way to do this, but it varies widely from computer to computer; see the other ???.f files for examples. It is best if you can define EXECUTION (CPU) time, but if you can't use REAL (Clock) time - one or both of these can be defined on EVERY computer. If all else fails and you cannot define any form of time - no problem - TART97 will still execute correctly if you just add a dummy routine to always define time as 0 ,

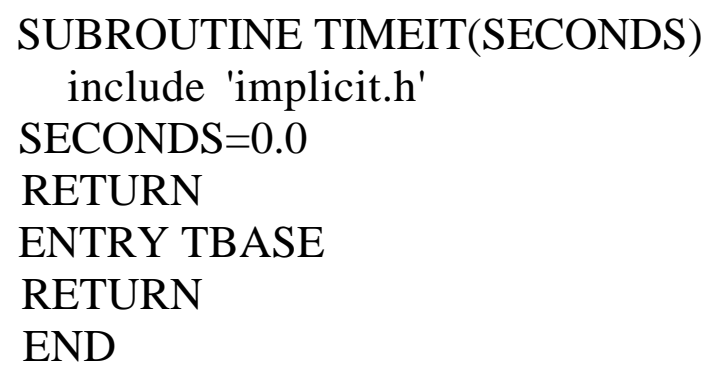

Note, this is not recommended, since you will not be able to time the execution of the codes; again, see more extensive remarks on timing at the end of this section.

\section{Subroutine DATEIT}

Define the time, data and computer in characters - 8 characters for each, for example,

12:00:00

$01 / 30 / 97$

SUN

Most computers have a way to do this, and it is useful documentation since it appears in your TART97 output file, which allows you to later determine exactly when any given problem was run; see the ???.f files for examples how this is done on a variety of computers. If your computer doesn't have a way to do this - no problem - just add a dummy routine to return any reasonable values that you want to appear in your output listing,

SUBROUTINE DATEIT(ARRAY)

CHARACTER *8 ARRAY(3) 


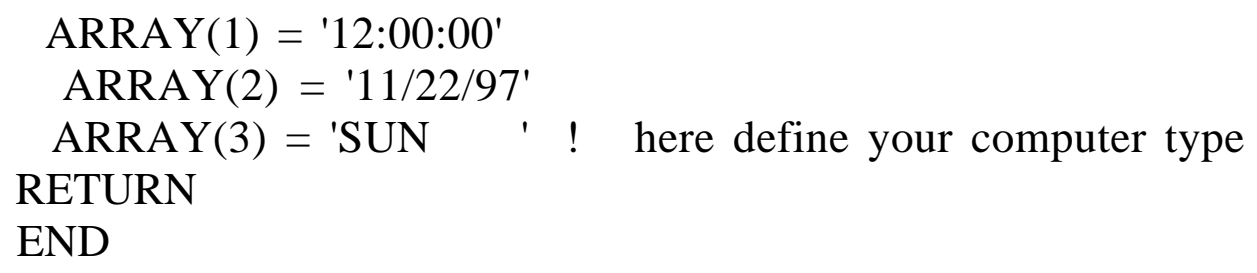

\section{That's it!!!!}

These are the only remaining computer dependent routines in TART97. Once you have defined an equivalent ???.f for these four routines for your computer you will be able to use the code.

If you do develop a ???.f for a different type of computer please return a copy of me - it will be included in future distributions of this code - which will make your work easier at that time.

You should next read the UNIX or CRAY portion of this installation manual and follow the steps to implement this system, at each step you can look inside the MAKEIT.BAT, etc. files to see what they are doing. You will see that they are merely compiling codes and executing them, and from the Makefiles for TART97, etc. you will be able to see which files to compile and load together. Once you understand what these files are doing you should be able to do the same thing on your computer.

\section{Timing Execution}

Most UNIX systems allow ETIME to be used to time execution, but there are a number of other routines - here's a list,

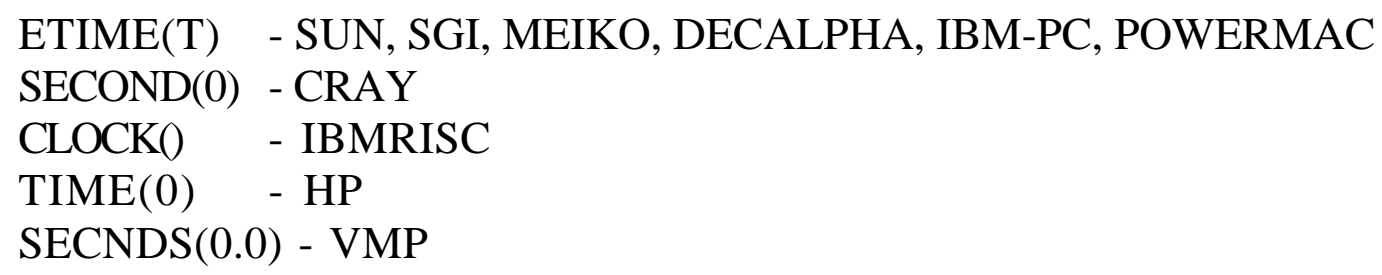

Individual routines are defined for all of these computers. If you have a different type of computer that doesn't use one of these routines I recommend that you use the SUN timing routine using ETIME and add your own defined ETIME routine at the end of the FORTRAN deck,

\section{FUNCTION ETIME(TARRAY)}




\section{DIMENSION TARRAY(2) \\ TIMENOW = ??????? \\ TARRAY $(1)=$ TIMENOW \\ TARRAY $(2)=0.0$ \\ ETIME $=$ TIMENOW \\ RETURN \\ END}

Where ??????? is the name of the routine on your system that defines time (execution or real time) in seconds - either from the start of execution or some fixed time - it doesn't matter, since TART97 will only use differences in time defined by successive calls to ETIME. 


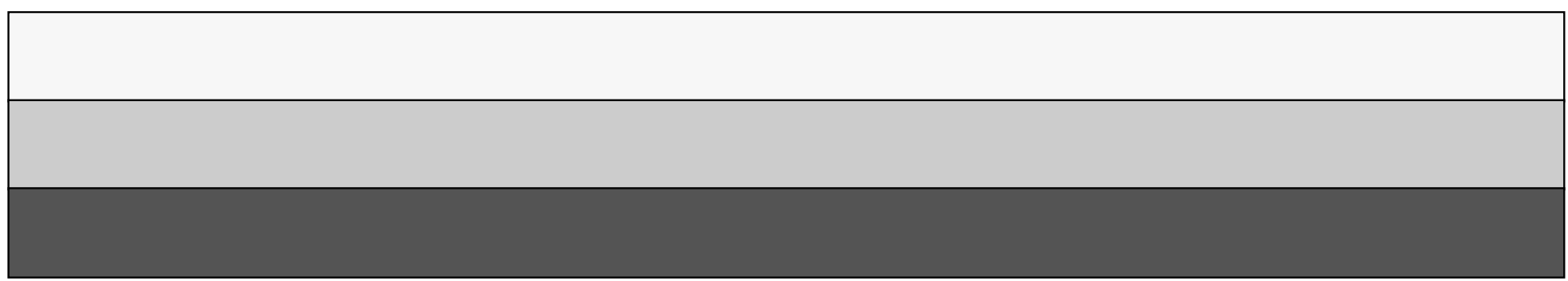

